\title{
Some aspects of the enzymatic basis of phytoremediation
}

\author{
G. Kvesitadze, ${ }^{1 *}$ M. Gordeziani, ${ }^{1}$ G. Khatisashvili, ${ }^{1}$ T. Sadunishvili ${ }^{1}$ and J. J. Ramsden ${ }^{2}$ \\ ${ }^{1}$ Durmishidze Institute of Biochemistry and Biotechnology, Academy of Sciences of Georgia \\ David Agmasheneblis Kheivani 10 km, 350059 Tbilisi, Georgia \\ ${ }^{2}$ Department of Biophysical Chemistry, Biozentrum, University of Basel, Switzerland
}

For the first time in the history of mankind, man has a realistic possibility of countering millenia of apparently selfish exploitation of nature by the application of scientific knowledge to devise effective remediation schemes. This review concerns the potential of phytoremediation, i.e. the use of plants to neutralize harmful toxic substances. The broad strategy of the plant when confronted by a xenobiotic is to oxidize it, thereby enabling its insertion into standard metabolic cycles (including its ultimate degradation carbon dioxide). Compounds which cannot be oxidized are rendered harmless by conjugation to hydrophilic compounds available within the cell, which not only decreases the toxicity of the xenobiotic, but facilitates its movement to depositories such as the vacuole. The oxidative enzymes act not only to oxidize xenobiotics, but also to hydroxylate them so that they can be conjugated. Hydroxylation is catalyzed by metalloenzymes: cytochrome P450-containing monooxygenases, peroxidases and copper-containing polyphenoloxidases. Cytochrome $\mathrm{P} 450$ is characterized by very low specificity, enabling it to treat an extraordinarily wide range of organic compounds. Under certain conditions plant microsomal cytochrome P450 loses its monooxygenase activity and becomes a peroxidase: this switching is an example of the sophisticated ability of plants to adapt to stress.

Keywords: conjugation, cytochrome P450, malate dehydrogenase, monooxygenases, oxidative enzymes, peroxidase, polyphenoloxidase, xenobiotics, ultrastructural reorganization

\section{INTRODUCTION}

The history of mankind is marked by unremitting and selfish exploitation of nature. Consequently, the end of the 20th century was characterized by widespread atmospheric pollution, accumulation of toxins in the soil and generally progressive deterioration of the environment. Indeed, the release into the atmosphere of carbon monoxide, carbon dioxide and associated pollutants from the extensive burning of fossil fuels is now on a scale large enough to destroy many kinds of organisms. Moreover, technical progress has been accompanied by the generation of many new chemicals; currently it is estimated that more than 300 million tons of various compounds are synthesized annually. Many of them, including herbicides, insecticides and acaricides, accumulate directly or indirectly in soil, water

*Author for correspondence.

Tel.: +995 32 958145; fax: +995 32 250604;

e-mail:kvesitadze@hotmail.com reservoirs and air. Because their rate of accumulation is more rapid than the capacity of our planet to remove these toxicants, their net effect on the environment is characterized by the appearance of defective forms of plants, microorganisms and animals.

In response to increased global pollution, progressively more attention is now being paid to the effective use of natural detoxifying agents. Foremost amongst these are microorganisms, which with their high transforming and degrading capabilities are successfully being used for the purification of sewage and soil. Plants are also capable of absorbing and metabolizing many different types of organic pollutants. Alongside their detoxificational abilities, plants have the added advantages of their unique photosynthetic capacity, participation in symbiotic nitrogen fixation, yield of economically important harvests, and an almost universal distribution: plants occupy over $45 \%$ of the world's landmass. Hence, plants play a pivotal rôle, both in maintaining a general ecological balance in nature and as natural remediators of man-made environmental pollution. This review focuses on the means by which plants metabolize pollutants. 


\section{UPTAKE}

Organic pollutants are absorbed by plants both from the air, and from the soil after being dissolved in water and taken up together with nutritional components $[1,2]$. The absorption of pollutants through roots and leaves differs: to get into the root, a substance must penetrate only through unsuberificated (i.e. not containing the reinforcing compound suberin) cell walls free of cuticle; but to get into the inner part of the leaf, a substance must penetrate either through the stomata or through the cuticle of the epidermis. The absorption of dissolved compounds by the plant is a controllable process. The rhizodermis of young roots absorbs foreign compounds by osmosis. This absorption depends greatly on such external factors as temperature and $\mathrm{pH}$ of the nutritional ambient and the soil. Other factors that significantly influence the penetration of toxicants into the plant are their molecular weights and their lipophilicities (hydrophobicities), principally the latter determining movement of organic pollutants across the plant cell membrane. After passage across the membrane, xenobiotics are distributed throughout the entire plant. What then is the further fate of the pollutant molecule?

\section{ELIMINATION: EXCRETION, OXIDATION AND CONJUGATION}

The penetration of organic toxicants into plant cells is almost invariably associated with the induction of significant changes to the normal ranges of parameters characterizing intracellular metabolic processes; each change represents one means by which the plant attempts to avoid the toxic action of the absorbed pollutant. Excretion provides the simplest method of eliminating an absorbed xenobiotic. Xenobiotics absorbed through the roots are only rarely excreted via the leaves since this process involves the often onerous additional steps of phytovolatilization $[3,4]$. Much more common is the excretion of toxicants absorbed by leaves via the root system. Nevertheless, when the concentration of toxicants is high only a small fraction (less than 5\%) is excreted in unchanged form, and full detoxification of organic compounds in plants is achieved by mineralization to carbon dioxide. Clearly, this process must be performed via oxidative reactions. The ability of a plant to intensively undertake these reactions determines its detoxificational potential.

If the plant lacks the potential to swiftly oxidize an absorbed toxicant, then conjugation of the toxicant with intracellular compounds and its subsequent deposition in vacuoles takes place. Conjugation is not only one of the main detoxificational mechanisms in higher plants but also acts to prevent adverse actions of toxicants on the informational, energetic or biosynthetic centres within plant cells. Hydrophilic compounds such as carbohydrates, organic and amino acids, peptides and proteins are enzymatically (by transferases [5]) conjugated with the xenobiotics, or primary products of their transformation, via their hydroxyl, carboxyl, amino or other functional groups. The result of these transformations is, firstly, to significantly decrease the toxicity of absorbed xenobiotics for the plant, and secondly to increase their hydrophilicity, resulting in enhanced mobility of the conjugates within the cytoplasm and facilitated deposition in the vacuoles (Fig. 1). Ether, ester, peptide and other easily hydrolyzable bonds participate in the formation of conjugates, creating a good basis for the dissociation of deposited compounds and their further extensive oxidation. Conjugation could be considered as the terminal act of phytoaccumulation and phytostabilization; the analogous process for phenolic compounds is their incorporation into lignin in inactive form, which thus serves as a vacuole-like but solid depository.
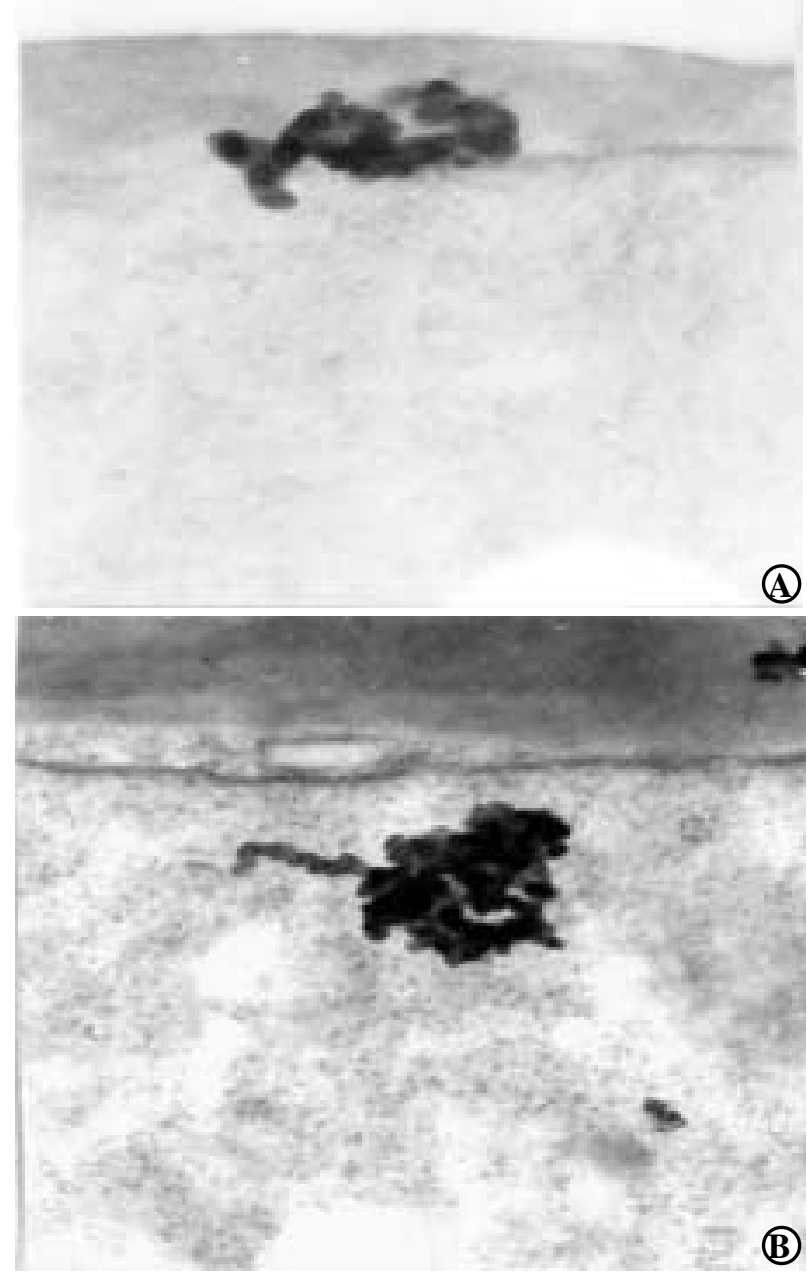

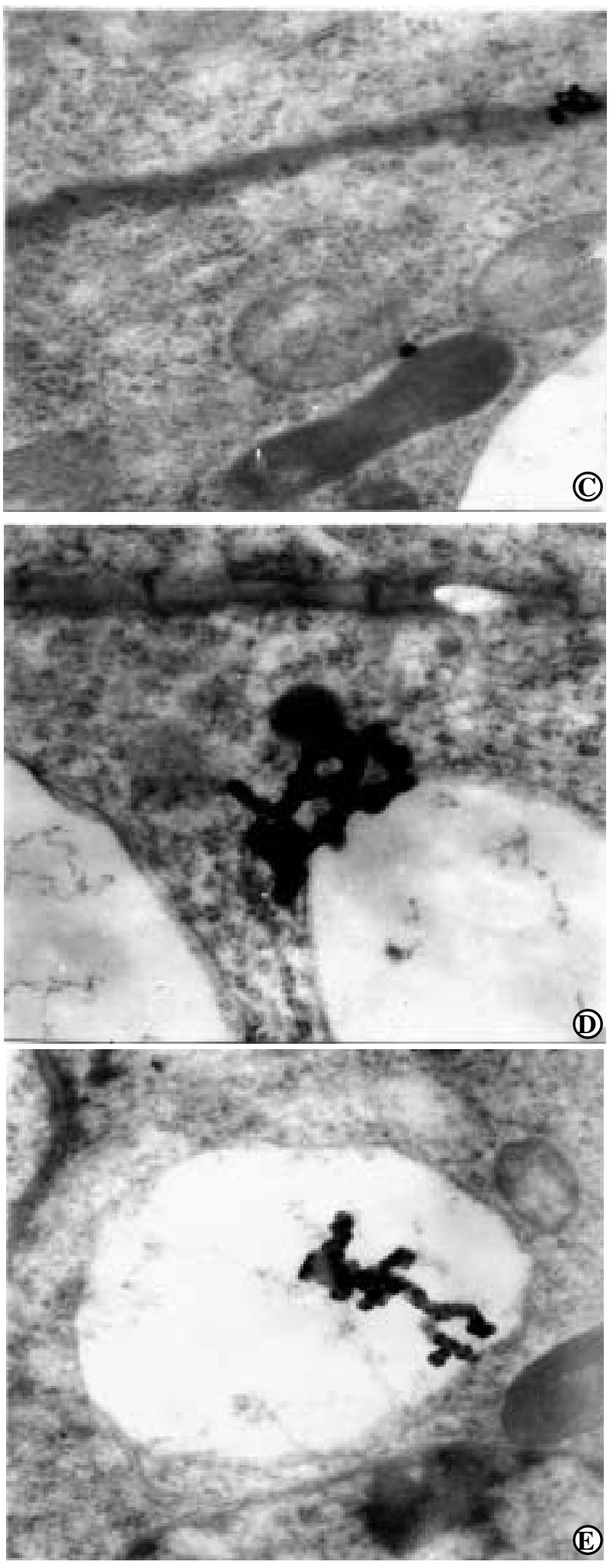

Figure 1. Electron micrographs showing the uptake and movement of ${ }^{14} \mathrm{C}$-labelled nitrobenzene in a maize root tip cell. The xenobiotic penetrates through the plasmalemma (A), moves to the cytoplasm (B), locates near membrane-associated organelles (C) and is thereafter translocated directly into vacuoles $(\mathrm{D}, \mathrm{E})$.

$(\mathrm{A}, \times 48000, \mathrm{~B}, \times 36000, \mathrm{C}, \times 28000, \mathrm{D}, \times 50000$, $\mathrm{E}, \times 30000)$

\section{CONJUGATION}

In order to carry out the oxidative degradation of toxicants, the plant cell, in addition to some ultrastructural reorganization, undergoes other intracellular changes including the formation of conjugates and their deposition in vacuoles. These processes imply the expenditure of energy, supplied as reducing equivalents from NADPH generating systems (photosynthesis, pentose cycle) in mitochondria, from freely available molecular oxygen, and from free radicals produced by the peroxidation of lipids.

Within our present state of knowledge the following aspects of the fate of toxicant conjugates within plant cells remain unclear: (i) their stabilities and half lives; (ii) the activities of toxicants after conjugate dissociation; (iii) the extents of oxidative degradation - in the context of conjugation - of different xenobiotics; and (iv) the physiological consequences for the plant itself of the conjugation of organic pollutants.

Changes in cell metabolism occur due to conjugate formation, especially where concentrations of toxicants are high. The excessive expenditure of cell energy associated with the creation of conjugates impairs cellular function. Vitally important endogenous metabolites are sequestered during the process of conjugate formation. On entering the cellular food chain, conjugates dissociate and may consequently release toxic components. The conjugation process can, therefore be considered as an intermediate stage resulting in the incomplete transformation of the toxic parts of xenobiotics by their temporary conjugation to endogenous metabolites.

\section{FUNCTIONALIZATION}

Often nonpolar toxicants do not contain the functional groups necessary for a conjugation reaction. In order to increase the reaction potential of these nonpolar compounds, incorporation of the necessary functional groups into the xenobiotic molecule is required. This process of functionalization increases the polarity of the whole molecule and is performed enzymatically by oxidation, reduction or hydrolysis. The resulting hydrophilic products are much more readily utilized by plant cells.

Functionalization requires a high activation energy and is the most severely rate-limiting reaction in the entire multistep process of intracellular toxicant transformation.

Hydroxylation is one of the most widely encounte- 
red functionalization reactions in plants and is effected by insertion of an oxygen molecule into a $\mathrm{C}-\mathrm{H}$ bond of the xenobiotic molecule [6,7]. The reaction requires the participation of active oxygen. In plants, hydroxylation is catalyzed by metalloenzymes: cytochrome $\mathrm{P} 450$-containing monooxygenases, peroxidases and polyphenoloxidases. The monooxygenase system is localized in the endoplasmic membrane and is a highly organized multienzyme complex. For activation of molecular oxygen this sytem exploits NADH (produced mainly in mitochondria) and NADPH(produced mainly in chloroplasts) reduced equivalents. The individual components of the monooxygenase system-NADPH-cytochrome P450reductase, cytochrome $\mathrm{b}_{5}$ and cytochrome $\mathrm{P} 450$ possess sufficient redox potential in order to apply molecular oxygen to the oxidation of organic compounds. The terminal electron acceptor, cytochrome P450, is a hemoprotein. The iron ion situated in the active site of this molecule binds molecular oxygen and the xenobiotic molecule activates oxygen by electrons received from the redox chain, and one of its atoms is then incorporated into the substrate molecule [8-11]. This reaction is presented in Scheme 1.

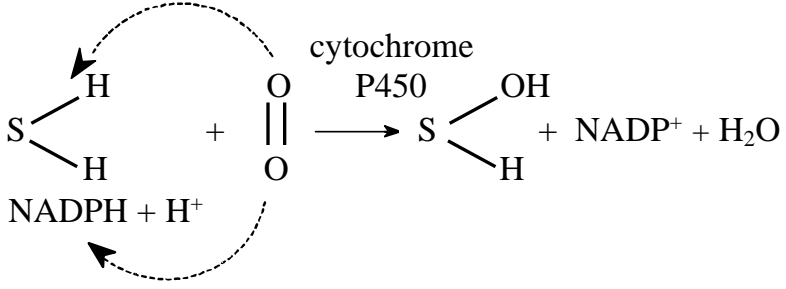

Scheme 1. S denotes substrate; the cosubstrate is NADPH.

According to Scheme 1, during monooxygenation one atom of the oxygen is reduced to water, i.e. the oxygenase participating in this reaction acquires the properties of a reductase; because of their ability to carry out this dual reaction these enzymes are known as oxidases having mixed function.

It should be borne in mind that functionalization alone is insufficient to render a xenobiotic nontoxic for its host.

\section{THE ENZYMES OF OXIDATION}

After functionalization of the xenobiotic in plants, during which its primary structure remains intact, extensive oxidative degradation takes place. This has been confirmed by numerous studies showing the inclusion of the transformed intermediates in the Krebs cycle, other cell processes, and the excretion of ${ }^{14} \mathrm{CO}_{2}$ following exposure to ${ }^{14} \mathrm{C}$-labelled xenobiotics $[1,2]$. Xenobiotic conjugates accumulated in vacuoles are highly likely to undergo extensive oxidation after their release. Hence, functionalization prepares a xenobiotic either for immediate further oxidation, or for conjugation, whereby oxidation is deferred until the eventual release of the compound.

In Scheme 2 below, a classical example of extensive oxidation of an organic pollutant in plants is shown: the full degradation of an aromatic carbon skeleton down to carbon dioxide. Muconic acid is the first aliphatic compound formed as a result of aromatic ring splitting, which is further oxidized to fumaric acid and then participates in the cell metabolism as a standard endogenous substrate.

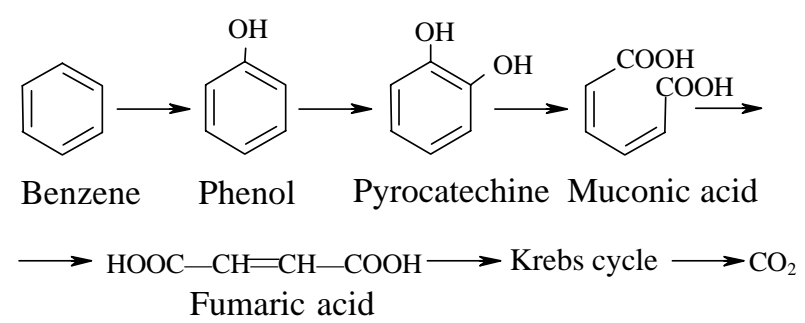

Scheme 2.

It is apparent that the importance and universality of plants in bioremediation lies in their ability to take up from the air, water and soil all types of organic pollutants and thereafter to oxidatively degrade them down to carbon dioxide. As the result of such transformation, all the carbon atoms of the toxicant acquire the ability of insertion in the pathways of biogenic migration, i.e. participation in the characteristic cycle of carbon circulation. This is the ultimate basis of the phytoremediational process.

All the important pathways of xenobiotic oxidation (mediated by iron and copper containing enzymes) operate in plant cells consecutively or simultaneously. The organization and functioning of the cytochrome P450-containing monooxygenase systems in procaryotic and eucaryotic organisms are distinguished by specialized features. Procaryotes contain soluble forms of this enzymatic system. In eucaryotes, the quinary structure of the hemoproteins is established by their incorporation into the endoplasmic membrane [14]. The classic example is liver cytochrome $\mathrm{P} 450$, which is readily incorporated into the membrane structure. The individual components of the monooxygenase system are positioned along the entire membrane. In this configuration they are in close contact with the lipid matrix, which at the same time appears to have a barrier function; therefore oxidative hydroxylation in microsomes is preceded by penetration of the xenobiotic through the membrane lipid layer. Formation of a catalytically active complex between cytochrome $\mathrm{P} 450$ and the xenobiotic determines its movement from the aqueous to the phospholipid phase. 
Plants possess a cytochrome P450-containing monooxygenase system built into the membrane. However, plant cells also contain soluble forms of the same enzyme, enabling them to significantly enhance their detoxifying capacity.

A unique feature of the monooxygenase terminal component, namely cytochrome P450, is its extremely low specificity. This confers on the molecule its universal capacity to hydroxylate organic compounds with widely differing chemical structures. Besides its low substrate specificity, the monooxygenase system requires the presence of nonpolar groups in a xenobiotic molecule. The transformation of ionized forms of xenobiotics in microsomes does not take place.

Plant peroxidase is a ubiquitous, catalytically versatile enzyme [15-17]. Its isozymes in green plants occur in the cell walls, the plasmalemma, the intracellular membrane system and in the cytoplasm. This hemoprotein contains carbohydrates (approximately $25 \%$ of the whole mass) that protect the enzyme from proteolytic degradation. The enzyme catalyzes the following reaction:

$$
\mathrm{RH}_{2}+\mathrm{XOOH} \longrightarrow \mathrm{R}+\mathrm{H}_{2} \mathrm{O}+\mathrm{XOH}
$$

where $\mathrm{RH}_{2}$ is the substrate subjected to oxidation and $\mathrm{XOOH}$ is the peroxide compound. Peroxidase furthermore plays an important rôle in metabolic processes in plant cells and its concentration increases under stress conditions. One of the key functions of plant peroxidases is to protect the cell from hydrogen peroxide generated as a result of oxidative reactions during photosynthesis, but the wide distribution of peroxidases in the cell enables the enzyme to oxidize a diverse spectrum of foreign compounds. For preliminary activation of oxygen, peroxidase does not need external energy since it uses its active form for xenobiotic hydroxylation. The enzyme is also characterized by low specificity and high affinity for xenobiotics of different chemical natures. This feature ensures the active participation of peroxidase in a wide variety of detoxificational processes.

Phenoloxidase is a copper containing protein, localized in the chloroplasts of higher plants [16]. The enzyme exists in multiple forms in active and latent conditions and catalyzes both the monooxygenase and oxygenase reactions (Scheme 3).

In addition to its main function of catalyzing the oxidation of phenolic compounds, phenoloxidase also actively participates in xenobiotic oxidation. In this process, depending on the structure of the substrate, both these activities of the enzyme are actively

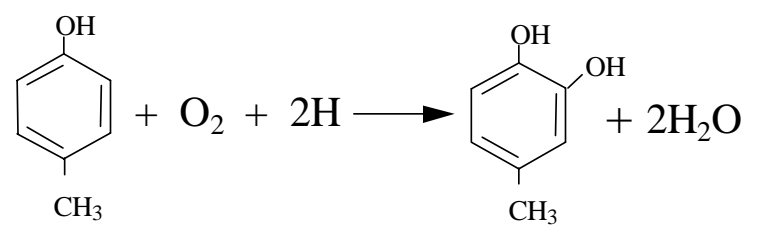

2

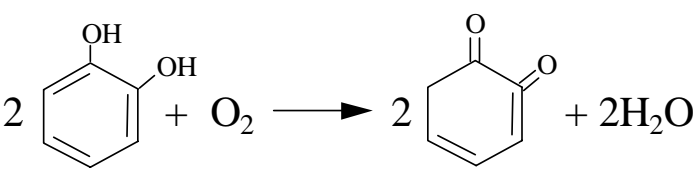

Scheme 3.

utilized. Phenoloxidase effects the oxidation of aromatic xenobiotics, their hydroxylation and further oxidation to quinone. If the xenobiotic subjected to oxidation is not a substrate for phenoloxidase, the enzyme oxidizes phenol by forming $o$-quinone, which then itself takes part in the xenobiotic oxidation.

\section{ULTRASTRUCTURAL REORGANIZATION}

Electrons for the activation of oxygen are obtained by cytochrome P450 from the respiratory chain $[12,13]$. To perform such electron transfer, close contacts between the mitochondrial and microsomal membranes are required. Indeed, after the action of a xenobiotic on a plant cell, the concentration of microsomal membranes around mitochondria and the establishment of close contacts between them are markedly enhanced (Fig. 2). Such ultrastructural reorganization indicates close coordination between the energetic (mitochondrial) and free electron transport (reticulum) systems, creating optimal conditions for the functioning of the oxidative

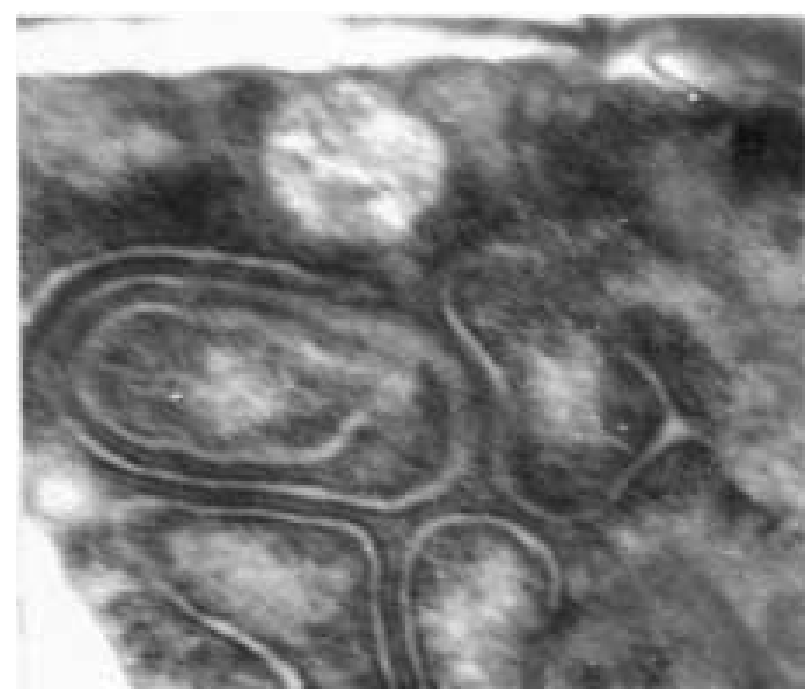

Fig 2. As a result of the intracellular action of xenobiotics close apposition between endoplasmic reticulum and mitochondria are often seen. The electron microgram shows the enrichment of endoplasmic reticulum around the mitochondria. $(\times 50000)$. 
enzymes. The main feature of this regulatory mechanism lies in the fact that the great majority of xenobiotics simultaneously appear to be substrates for cytochrome $\mathrm{P} 450$ and inhibitors of the respiratory chain. Mitochondrial oxidation is inhibited when the microsomal hydroxylase system is saturated by a high concentration of xenobiotic. Contact between mitochondria and microsomes occurs as a consequence of the ability of mitochondria to move freely in the cytosol and accumulate at sites of energy deficiency. The energy supply for these cellular organelles can be provided both by ATP and electrons with the requisite level of energy. It is therefore clear that the consumption of electrons provides the main reason for the close juxtapositioning of mitochondrial and microsomal membranes in plant cells affected by xenobiotics.

\section{ENZYME PLASTICITY}

The cytochrome $\mathrm{P} 450$-containing monooxygenases catalyze more then 20 different biosynthetic reactions in a plant cell and constitute the key enzyme group in the synthesis of phenolic compounds [9]. By decreasing its biosynthetic activity this enzyme participates in the hydroxylation of xenobiotics. The switch of an enzyme from biosynthesis to detoxification is determined by the polarity of the xenobiotic; the more hydrophobic the xenobiotic, the higher its affinity for P450, the more universal the switch, and the faster the process of oxidation [18]. After penetration of the xenobiotic, typically $80 \%$ of these P450 enzymes operate to detoxify. In essence, the switch is actuated by the superior affinity of the xenobiotic for the enzyme compared to its natural subshates.

The switch of the enzymes participating in the detoxificational processes is accompanied by the induction of monooxygenases, peroxidases and phenoloxidases. These enzymes are localized in those compartments (cell wall, plasmalemma, glycocalyx, vacuole, tonoplast, endoplasmic reticulum) where the oxidative degradation of toxicants is carried out (Fig. 3).

The polarity of the intermediates formed as a result of the first step of xenobiotic oxidation significantly determines their subsequent fate. When the intermediates formed are aggressive free radicals, the transformation of monooxygenase to peroxidase takes place.

Under certain conditions plant microsomal cytochrome P450 loses monooxygenase activity and acquires that of peroxidase $[18,19]$. These conditions include:

(i) plant aging: in 7 day old etiolated seedling microsomes the quantity of cytochrome $\mathrm{P} 450$ and
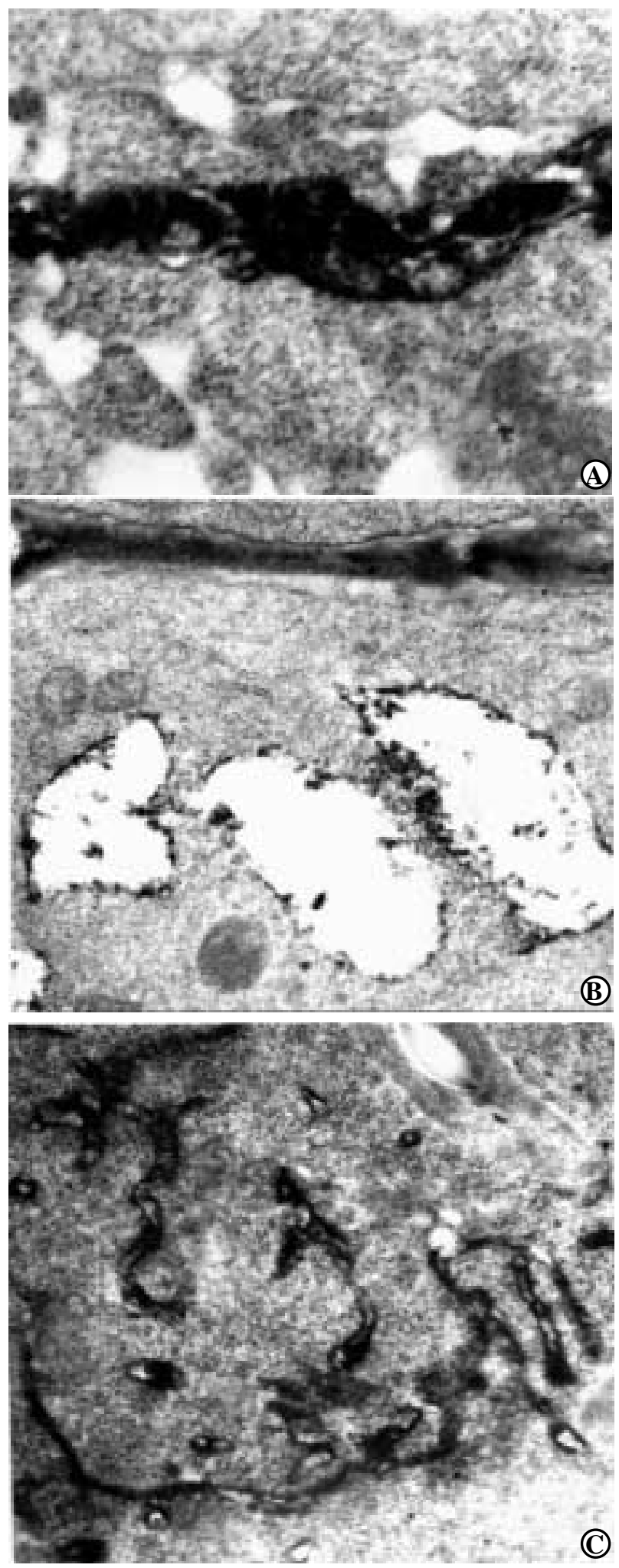

Figure 3. The induction of oxidative enzymes by xenobiotic activation is most evident in the case of peroxidase. After exposure to nitrobenzene large quantities of peroxidase cytochemical reaction product is observed in the cell wall, visible as an electron-dense precipitate. Plasmalemma and glycocalyx (A,B), vacuole and tonoplasts (B), and endoplasmic reticulum (C).

(A, $\times 30000, \mathrm{~B}, \times 30000, \mathrm{C}, \times 20000)$. 
correspondingly the microsomal monooxygenase activity is at a maximum. During the following days the cytochrome $\mathrm{P} 450$ is converted to P420, and simultaneously the monooxygenase activity decreases and peroxidase activity increases. In 14 day old seedlings the peroxidase activity is enhanced five- to six-fold and the ratio of cytochromes P420/P450 is 30 to 32 fold;

(ii) constant aeration of the microsomal fraction for 120 min results in a twofold increase of peroxidase activity and a tenfold increase of the cytochromes $\mathrm{P} 420 / \mathrm{P} 450$ ratio;

(iii) during the process some substrates are oxidized by cytochrome $\mathrm{P} 450$. The results indicated above provides grounds for assuming that cytochrome P450 can act either as a monooxygenase or as a peroxidase during xenobiotic oxidative degradation. The plant cell is, therefore, equipped with an extremely adaptable enzyme, able simultaneously to induce both oxidation of xenobiotics and also the elimination of hydrogen peroxide. Indeed, the participation of oxidized cytochrome $\mathrm{P} 450$ in peroxidase reactions is of crucial importance and enables both NADH and NADPH to act as sources of hydrogen. The interaction of cytochrome P450 with hydroperoxides forms an analogue with peroxidase, in which the heme iron is in a high oxidation state (complex I of peroxidase) [20] (Scheme 4).

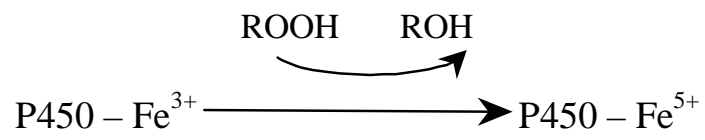

\section{NADH, NADPH}

Scheme 4.

The process of oxidation by hydrogen peroxides induces the destruction of cytochrome $b_{5}$, resulting in damage the monooxygenase systems. The RO and $\mathrm{RO}_{2}{ }^{\circ}$ radicals formed during peroxidation act on the cytochrome $\mathrm{P} 450$ porphyrin nucleus and degrade the apoenzyme.

Analogously to cytochrome P450, which carries out oxidation by monooxygenase and peroxidase mechanisms, peroxidase also can catalyze a non characteristic phenoloxidase reaction [16].

Thus, in plants the oxidation of xenobiotics can be performed by enzymes of different types or by one enzyme acting in different ways. By the interplay of these enzymatic mechanisms the plant cell acquires significant power to oxidatively degrade a wide spectrum of organic pollutants.

\section{CONCLUSIONS}

After the penetration of a toxicant through the cell wall, all the cell's energetic and metabolic potential is directed towards the degradation of the toxic compound. Firstly the oxidative enzymatic systems are mobilized. Penetration of xenobiotic appears to be the signal for the recruitment of enzymes participating in the biosynthetic detoxification reactions. One example of how this signalling can occur at the molecular level of detail is provided by the detailed investigation of the response of the procaryote Streptomyces lividans to the antibiotic streptomycin [21].

The penetration of organic toxicants into plant cells causes significant changes across the whole range of intracellular metabolic processes. This phenomenon is in full accord with biochemical logic and should occasion no surprise: detoxification requires the full mobilization of the internal metabolic potential of the plant cell. It is fighting against strange and at the same time toxic compounds with all accessible means. This manifests itself firstly in activation, or more precisely in the induction of synthesis, of those enzyme systems which directly participate in xenobiotic detoxification. These are enzymes participating in the following processes: (i) conjugation of xenobiotics with endogenous compounds; and (ii) oxidative degradation of xenobiotics. During these processes a significant amount of internal cell endogenous energy as well as vitally important compounds are expended. Presumably the great majority of cell enzymes are mobilized and somehow involved; conceptually it is the vital equivalent of le Chatelier's principle in operation (and cf. the response of procaryotes to starvation stress [22].

There are quite a few examples indicating that the enzymes participating in catabolic processes, namely in energy accumulation (Krebs cycle), are also affected by xenobiotic detoxification For instance, inhibition of glutamine synthetase activity (up to 50\%) and a simultaneous increase of glutamate dehydrogenase activity $(40 \%)$ in alfalfa results exposure to phosphinotricine [23].

Our investigations are revealing from the current correlation between the penetration of xenobiotics into plant cells and changes in the activities of the enzymes participating in energy and nitrogen metabolism. For example, an increase of malate- and glutamate dehydrogenase activities was demonstrated under the influence of different organic toxicants. It should be emphasized that the nature of this correlation is highly affected by the concentration of xenobiotics which have penetrated into the cell. 
Thus, as a result of toxicant penetration into plant cells, in addition to detoxification via oxidative degradation (the main detoxificational pathway) and conjugation, other processes which may be considered uncharacteristic for plant cells are taking place, notably ultrastructural changes and intensified activity of the enzymes participating in the Krebs cycle, due to an increased "fuel" supply.

Undoubtedly, the determination of the precise detoxification potential of plants and the elucidation of biochemical mechanisms of phytoremediation depend both upon the establishment of a sound theoretical base and the accumulation of more experimental data. However even the existing level of knowledge allows the creation of qualitatively new phytoecological technologies for remediation and long term protection of the environment. The foundation for such phytotechnologies could be based on the following consideration: because of their adaptation to diverse ecological niches, plants differ significantly from one another in their capacity to assimilate and metabolize toxicants. The differences between the detoxificational abilities of different plant varieties can easily exceed three orders of magnitude. On the basis of comprehensively accumulated data on these abilities, the planting of specially selected greenery near and along hotbeds of pollution (motorways, railways, oil pipelines, chemical and metallurgical plants, etc.) would minimize the flow of toxicants from them and improve the local ecological situation. It follows, therefore, that the detoxificational potential of plants should be an extremely important criterion for the proper specification and selection of agricultural and decorative, annual and perennial, wild and cultivated plants. International recognition and wide practical application of phytoremediation and phytoprotection as a base for ecotechnologies could significantly improve the ecological situation of the entire planet.

\section{REFERENCES}

1. Korte, F., Kvesitadze G., Ugrekhelidze D., Gordeziani, M., Khatisashvili, G. Buadze, O. Zaalishvili, G., Coulston, F. Review: organic toxicants and plants. Ecotoxicology Environmental Safety. 47 (2000) 1-26.

2. Zaalishvili, G., Khatisashvili, G., Ugrekhelidze D., Gordeziani, M., Kvesitadze G. (2000) Plant potential for detoxification (review). Appl. Biochem. Microbiol. 36, 443-451.

3. Arthur, E.L., Coats, J.R. Phytoremediation. In: Pesticide Remediation in Soil and Water. (ed. P. Kearney \& T. Roberts), pp. 251-283.New
York:Wiley (1998).

4. Salt, D.E., Smoth, R.D., Raskin, I. Phytoremediation. A. Rev. Plant Physiol. Mol. Biol. 49 (1998) 643-668.

5. Jakoby, W.B., Zeigler, D.M. The enzymes of detoxication. J. Biol. Chem. 265 (1990) 2071520718.

6. Sandermann, H. Plant metabolism of xenobiotics. Trends Biochem. Sci. 17 (1992) 82-84.

7. Sandermann, H. Higher plant metabolism of xenobiotics: the "green liver" concept. Pharmacogenetics 4 (1994) 225-241.

8. Archakov, A.J., Bachmanova, G.I. Cytochrome P450 and Active Oxygen. London: Taylor \& Francis (1990).

9. Durst, F. Biochemistry and physiology of plant cytochrome P450. In: Frontiers in Biotransformation. Berlin: Akademie Verlag, 4 (1991) 191-232.

10. Durst, F., Benveniste, I., Lesot, A.A., Salaün, J.-P., Werck-Reichhart, D. Induction of plant cytochrome P-450. In: Regulation of Enzymatic Systems Detoxifying Xenobiotics in Plants, pp.19-34 Dordrecht, Kluwer (1997) .

11. Schuler, M.A. Plant cytochrome P450 monooxygenases. Crit. Rev. Plant Sci. 15 (1996) 235284.

12. Cinti, D.L., Schenckman, J.B. Hepatic organelle interaction. I. Spectral investigation during drug biotransformation. Molec. Pharmacol. 8 (1972) 333-338.

13. Gordeziani, M., Khatisashvili, G., Ananiashvili, T., Varazashvili, T., Kurashvili, M., Kvesitadze, G., Tkhelidze P. Energetic significance of plant monooxygenase individual components participating in xenobiotic degradation. Int. Biodeterioration and Biodegradation 44 (1999) 49-54.

14. Archakov, A.I. Oxygenases of Biological Membranes. In: XXXVII Bakhovskie Chtenia, pp. 1-26. Moskow: Nauka (1983) (in Russian).

15. Stiborova, M., Anzenbacher, P. What are the principal enzymes oxidizing the xenobiotics in plants: cytochrome P-450 or peroxidase? Gen. Physiol. 10 (1991) 209-216.

16. Zeigel, B.Z. Plant peroxidases-an organismic perspective. Plant Growth Regulation 12 (1993) 303-312.

17. Laurent, F.M.G. Cloroaniline peroxidation by soybean peroxidases. Pesticide Sci. 40 (1994) 2530.

18. Khatisashvili, G., Gordeziani, M., Kvesitadze, G., Korte, F. Plant monooxygenases: participation in xenobiotic oxidation. Ecotoxicology Environmental Safety 36 (1997) 118-122. 
19. Khatisashvili, G., Kurashvili, M., Gordeziani, M., Kvesitadze, G. Monooxygenase and peroxidase pathways of xenobiotics detoxication in higher plants. Fresenius Environmental Bull. 2 (1993) 103-108.

20. Metelitsa, D.I. The Activation of Oxygen by Enzymatic Systems. Moscow: Nauka. (1982) (in Russian).

21. Chiu, M.L., Viollier, P.H., Katoh, T., Ramsden, J.J., Thompson, C.J. Ligand-induced changes in the Streptomyces lividans TipAL protein imply an alternative mechanism of transcriptional activation for MerR-like proteins. 22. Vohradský, J. and Ramsden, J.J. Genome resource utilization during procaryotic development. FASEB J. 15 (2001) 2054-2056 (online: 10.1096/ fj.00-0889fje).

23. Lacuesta, M., Gonzales-Moro, B., Gonzales, G., Aparicio-Tejo, P., Munioz-Rueda, A. Effect of phosphinothricin (glufosinate) on activities of glutamine synthetase and glutamate dehydrogenase in Medicago sativa L. J. Plant Physiol. 134 (1989) 304-307. 